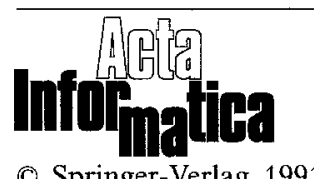

C Springer-Verlag 1991

\title{
Correction to \\ Null values in nested relational databases by M.A. Roth, H.F. Korth, and A. Silberschatz
}

\author{
M. Levene ${ }^{1}$ and G. Loizou ${ }^{2}$ \\ ${ }^{1}$ Centre for Information Technology, Department of Computer Science, \\ University College London, Gower Street, London, WC1E 6BT, UK \\ ${ }^{2}$ Department of Computer Science, Birkbeck College, Malet Street, London, WC1E 7HX, UK
}

\begin{abstract}
We show that the extended projection of a nested relation in Partitioned Normal Form (PNF) is not a precise generalization of standard projection with respect to unnesting and PNF possibility function POSS*.
\end{abstract}

The extended projection of a nested relation, $r$, over relation scheme, $R$, on attributes, $X^{*}$, denoted by $\prod_{X^{*}}^{e}(r)$, is given in Definition 22 of [1] by

$$
\prod_{X^{*}}^{e}(r)=\bigcup_{t \in \Pi_{X *}(r)}^{e}(t)
$$

where $\bigcup^{e}$ is the extended union operator of Definition 17 therein.

It was claimed in [1], Proposition 5 thereof, that the extended projection operator, $\prod^{e}$, is a precise generalization of the standard projection operator, $\prod$, with respect to unnesting, i.e.,

$$
\mu^{*}\left(\prod_{X^{*}}^{e}(r)\right)=\prod_{X}\left(\mu^{*}(r)\right)
$$

where $X$ is the set of zero order attributes corresponding to $X^{*}$ of Eq. (1).

We now proceed to show that the said Proposition 5 is, in fact, false by exhibiting a counterexample.

Lemma 1 Extended projection is not a precise generalization of the standard projection operator with respect to unnesting, i.e., Eq. (2) is false.

Proof. In order to prove the lemma we need to show that there exists a nested relation, $r$, over relation scheme, $R$, such that for a set of attributes, $X^{*}$, and the set of zero order attributes, $X$, corresponding to $X^{*}$, the following equation holds:

$$
\mu^{*}\left(\prod_{X^{*}}^{e}(r)\right) \neq \prod_{X}\left(\mu^{*}(r)\right)
$$


Now, let $r$ be the nested relation, over $R=\left(A, B^{*}\right)$, where $B^{*}=\left(B, C^{*}, D^{*}\right), C^{*}$ $=(C)$ and $D^{*}=(D)$, as shown in Fig. 1. It can easily be verified that the nested relation $r$ is in PNF, as given in Definition 1 [1]. That is, $A \rightarrow B^{*}$ holds in $r$, and also that $B \rightarrow C^{*}$ and $B \rightarrow D^{*}$ hold in the projections of the first and second tuples of $r$, over $\left\{B, C^{*}, D^{*}\right\}$.

\begin{tabular}{llll}
\hline$A$ & $B^{*}$ & & \\
\cline { 2 - 4 } & $B$ & $C^{*}$ & $D^{*}$ \\
& & $C$ & $D$ \\
\hline$a_{1}$ & $b_{1}$ & $c_{1}$ & $d_{1}$ \\
$a_{2}$ & $b_{1}$ & $c_{2}$ & $d_{2}$ \\
\hline
\end{tabular}

Fig. 1. The nested relation, $r$, over $R$

To conclude the proof of the lemma, let $r_{1}=\mu^{*}\left(\prod_{B^{*}}^{e}(r)\right)$ be the 1 NF relation, over $\{B, C, D\}$, shown in Fig. 2, and let $r_{2}=\prod_{\{B, C, D\}}\left(\mu^{*}(r)\right)$ be the $1 \mathrm{NF}$ relation over $\{B, C, D\}$, shown in Fig. 3. It follows that the extended projection operator, $\prod^{e}$, is not a precise generalization of the standard projection operator, $\prod$, since $r_{1} \neq r_{2}$.

\begin{tabular}{lll}
\hline$B$ & $C$ & $D$ \\
\hline$b_{1}$ & $c_{1}$ & $d_{1}$ \\
$b_{1}$ & $c_{1}$ & $d_{2}$ \\
$b_{1}$ & $c_{2}$ & $d_{1}$ \\
$b_{1}$ & $c_{2}$ & $d_{2}$ \\
\hline
\end{tabular}

Fig. 2. The $1 \mathrm{NF}$ relation, $\mu^{*}\left(\prod_{B^{*}}^{e}(r)\right)$, over $\{B, C, D\}$

We note that the impreciseness of the extended projection with respect to unnesting is due to the fact that the extended union operator, $U^{e}$, is not a precise extended operator with respect to unnesting as was shown in Fig. 5 of [1]. Furthermore, the extended union was used in the definition of the extended projection operator, and thus it is natural to question its preciseness.

\begin{tabular}{lll}
\hline$B$ & $C$ & $D$ \\
\hline$b_{1}$ & $c_{1}$ & $d_{1}$ \\
$b_{1}$ & $c_{2}$ & $d_{2}$ \\
\hline
\end{tabular}

Fig. 3. The 1NF relation, $\prod_{\{B, C, D\}}\left(\mu^{*}(r)\right)$, over $\{B, C, D\}$ 
As a consequence of Lemma 1, Proposition 11 of [1], which claims that the null-extended projection operator, denoted by $\prod^{e l}$, and given in Definition 28 therein, is a precise generalization of standard projection with respect to unnesting and PNF possibility function POSS*, is also false. An example to illustrate this fact is shown in Figs. 4, 5 and 6, where $r^{\prime}$ is the nested relation in PNF, shown in Fig. 4, with null in $r^{\prime}$ representing a null value. Let $\mu^{*}\left(\prod_{B^{*}}^{e l}\left(r^{\prime}\right)\right)$, over $\{B, C, D\}$, be the $1 \mathrm{NF}$ relation shown in Fig. 5 and let $\prod_{\{B, C, D\}}\left(\mu^{*}\left(r^{\prime}\right)\right)$, over $\{B, C, D\}$, be the $1 \mathrm{NF}$ relation shown in Fig. 6 . Then, it can easily be verified that equation (4) holds, thus proving our claim that null-extended projection is not a precise generalization with respect to unnesting and PNF possibility function POSS*. That is,

$$
\begin{aligned}
& \operatorname{POSS}^{*}\left(\mu^{*}\left(\prod_{B^{*}}^{e l}\left(r^{\prime}\right)\right)\right) \prod_{\{B, C, D\}}\left(\operatorname{POSS}^{*}\left(\mu^{*}\left(r^{\prime}\right)\right)\right) . \\
& \text { A } \quad B^{*} \\
& B \quad C^{*} \quad D^{*} \\
& \begin{array}{rrr}
C & D \\
\hline
\end{array} \\
& \begin{array}{llll}
\hline a_{1} & b_{1} & \text { null } & d_{1} \\
a_{2} & b_{1} & c_{1} & \text { null } \\
\hline
\end{array}
\end{aligned}
$$

Fig. 4. The nested relation, $r^{\prime}$, over $R$

\begin{tabular}{lll}
\hline$B$ & $C$ & $D$ \\
\hline$b_{1}$ & $c_{1}$ & $d_{1}$ \\
\hline
\end{tabular}

Fig. 5. The 1NF relation, $\mu^{*}\left(\prod_{B^{*}}^{e l}\left(r^{\prime}\right)\right)$, over $\{B, C, D\}$

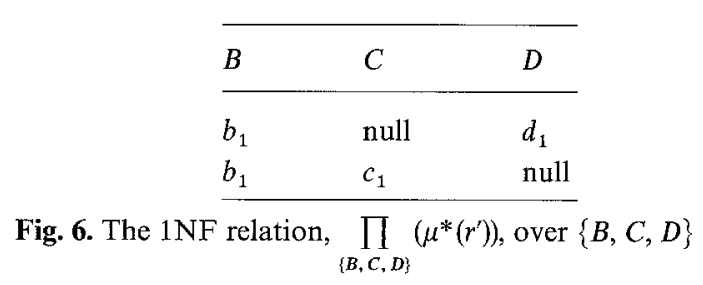

Finally, we observe that in order that the extended projection be precise it is necessary to define a decomposition projection operator, similar to the decomposition union of Definition 17 [1], which takes into account the multi-valued dependencies, or equivalently, the join dependency, which is produced by the nested structure over the set of projected attributes, $X^{*}$.

\section{References}

1. Roth, M.A., Korth, H.F., Silbersatz, A.: Null values in nested relational databases. Acta Inf. 26, 615-642 (1989) 